\title{
Disease Response Time Point Reference
}

National Cancer Institute

\section{Source}

National Cancer Institute. Disease Response Time Point Reference. NCI Thesaurus.

Code C162130.

The point in time that acts as a fixed reference point to a disease response assessment. 